\title{
Compared to What? Is BMI Associated with Histopathological Changes in Laparoscopic Sleeve Gastrectomy Specimens?
}

\author{
Tamer Saafan $^{1} \cdot$ Walid El Ansari ${ }^{2,3,4}$ (1) Moataz Bashah ${ }^{1,5}$
}

Published online: 15 April 2019

(C) The Author(s) 2019

\begin{abstract}
Background Obesity is a risk for many different cancers. Laparoscopic sleeve gastrectomy (LSG) is common, and benign or premalignant histopathology types are reported in the removed gastric specimens. We assessed whether higher BMI was associated with certain benign or pre-malignant histopathological changes.

Method Retrospective chart review of all primary LSG patients $(N=1555)$. Demographic, clinical, and LSG histopathology data were retrieved. BMI of patients with specific benign or pre-malignant conditions in their gastric specimens was compared with the BMI of the rest of the patients with abnormal histopathology specimens and also compared with the BMI of patients with normal control specimens. Results Females comprised 70\% of the patients. Mean BMI were 46.3 (females) and 48 (males). Normal LSG specimens comprised 52\%. Most common abnormal histopathologies were chronic inactive gastritis (33\%), chronic active gastritis (6.8\%), follicular gastritis (2.7\%), lymphoid aggregates (2.2\%), intestinal metaplasia (1.4\%) and GIST (0.7\%). After controlling for confounders (age, gender, H. pylori, diabetes mellitus type 2, hypertension), no significant association was observed between the BMI of patients with specific benign or pre-malignant histopathology compared with the BMI of the rest of the patients with abnormal histopathologies and compared to the BMI of patients with normal histopathologies.

Conclusion When confounders were taken into account, there appeared no significant associations between the BMI of patients with specific benign or pre-malignant histopathology compared with the BMI of the rest of the patients with abnormal histopathologies and compared to the BMI of patients with normal histopathologies of their gastric specimens. There was a very weak correlation between BMI and other covariates.
\end{abstract}

Keywords Laparoscopic sleeve gastrectomy $\cdot$ LSG $\cdot$ Histopathologies · BMI $\cdot$ Pre-malignant histopathology $\cdot$ Benign histopathology

\section{Introduction}

Bariatric procedures are common worldwide as a result of the obesity epidemic [1,2], and laparoscopic sleeve gastrectomy

Walid El Ansari

welansari9@gmail.com

1 Department of Bariatric Surgery, Hamad General Hospital, Hamad Medical Corporation, Doha, Qatar

2 Department of Surgery, Hamad General Hospital, Hamad Medical Corporation, PO Box 3050, Doha, Qatar

3 College of Medicine, Qatar University, Doha, Qatar

4 School of Health and Education, University of Skövde, Skövde, Sweden

5 Weill Cornell Medicine, Doha, Qatar
(LSG) is a widely practised procedure. Obese patients are at a higher risk of many cancers, e.g. endometrial and gastric cancer, oesophageal adenocarcinoma, as well as liver, kidney, and colorectal cancers [3-10]. LSG histopathology specimens may contain pre-malignant conditions such as follicular gastritis, lymphoid aggregate, intestinal metaplasia, gastrointestinal stromal tumour (GIST), atrophic chronic gastritis, autoimmune gastritis and dysplastic neuroendocrine nodule [10-16]. However, despite that obese patients are at higher risk of many cancers and despite the increased risk of pre-malignant conditions in LSG histopathology specimens, the literature suggests that very sparse research has been undertaken to explore the associations between BMI and histopathological changes identified in the gastric specimens of LSG patients [10, 17-19]. It is important to scrutinise the relationship between BMI and different histopathologies in morbidly obese patients undergoing LSG in order to assess whether higher BMI 
patients may be associated with higher rate of pre-malignant histopathologies and thus, the possibility that patients with higher BMI may need additional pre-operative workup.

Therefore, the aim of the current study was to examine, among LSG patients, the relationship between BMI and different histopathologies identified in the removed gastric specimens. The study sought to answer five specific questions:

1. Is the BMI of patients with given abnormal specimens (whether benign or pre-malignant) different from the BMI of controls with all abnormal specimens other than the given one?;

2. Is the BMI of patients with abnormal specimens (whether benign or pre-malignant) different from the BMI of controls with normal specimens?;

3. Is the BMI of patients with pre-malignant specimens different from the BMI of controls with normal specimens?;

4. Is the BMI of patients with benign specimens different from the BMI of controls with normal specimens?; and

5. Is the BMI of patients with benign specimens different from the BMI of controls with abnormal pre-malignant specimens?

Table 1 depicts the five research questions that the study sought to answer, along with the cases and controls employed for each question.

\section{Materials and Methods}

\section{Ethics and Sample}

The Medical Research Center at Hamad Medical Corporation approved the study protocol (Proposal No. 16202/16). The study was undertaken at Hamad General Hospital (HGH), the largest hospital in Qatar. We retrospectively analysed the demographic, clinical, and histopathologic data of patients who had undergone primary LSG at HGH from February 2011 to July $2014(n=1555)$.

\section{Procedures and Data Collection}

During this period, every LSG patient had pre-operative esophagogastroduodenoscopy routinely, in accordance with EAES guidelines and CLO test routinely, to detect Helicobacter pylori (H. pylori), where positive patients were given the standard triple therapy: amoxicillin and clarithromycin ( 2 weeks) and proton pump inhibitor (2 months). Our histopathology department examined all gastric LSG specimens macro- and microscopically and specified a diagnosis. Clinical data [age, gender, H. pylori, hypertension (HTN), type 2 diabetes (DM2)] and the findings of the histopathology specimens were extracted from the electronic medical records. The abnormal histopathologies were categorised into benign and pre-malignant histopathologies.

\section{Statistical Analysis}

Statistical analyses were done using statistical packages SPSS 22.0 (SPSS Inc. Chicago, IL) and Epi Info 2000 (Centers for Disease Control and Prevention, Atlanta, GA). A two-sided $p$ value $<0.05$ was considered statistically significant. Descriptive statistics summarised all demographic, anthropometric, clinical and other related characteristics of the participants. Normally distributed data and results were reported as mean and standard deviation (SD); the remaining results were reported with as median and interquartile range (IQR). Categorical data were summarised using frequencies and percentages. The primary outcome variable was to assess the relationship between BMI and different benign and premalignant histopathologies. Chi-square $\left(\chi^{2}\right)$ test and/or Fisher Exact test as appropriate assessed associations between two or more qualitative variables (such as gender, comorbidities etc. with BMI). Unpaired ' $t$ ' or Mann Whitney $U$ test as appropriate assessed quantitative data between the two independent groups. The relationship between BMI and other variables (age, gender, H. pylori, HTN, DM2 and different histopathologies) were assessed using Pearson's/ Spearman's correlation coefficients. Further, we applied linear

Table 1 Research questions, cases and controls

1. Is the BMI of patients with given abnormal specimens different from the BMI of controls with all abnormal specimens other than the given one?

Cases: Individual type of abnormal specimens

Controls: All abnormal specimens other than given one

2. Is the BMI of patients with abnormal specimens different from the BMI of controls with normal specimens?

Cases: Individual type of abnormal specimens

Controls: All normal histopathology specimens

3. Is the BMI of patients with abnormal pre-malignant specimens different from the BMI of controls with normal specimens?

Cases: All abnormal pre-malignant specimens combined

Controls: All normal histopathology specimens

4. Is the BMI of patients with abnormal benign specimens different from the BMI of controls with normal specimens?

Cases: All abnormal benign specimens combined

Controls: All normal histopathology specimens

5. Is the BMI of patients with abnormal benign specimens different from the BMI of controls with abnormal pre-malignant specimens?

Cases: All abnormal benign specimens combined

Controls: All abnormal pre-malignant specimens combined 
regression methods to assess the relationship between BMI and all independent covariates stated above, and we estimated the regression coefficient from the linear regression model considering BMI as the outcome variable of interest.

\section{Results}

Table 2 shows the demographic, anthropometric and clinical characteristics of LSG patients $(N=1555)$. About $70 \%$ of the sample were females. Mean BMI and age for females and males were 46.3 and 48 years, and 36 and 35.3 years, respectively. H. pylori, HTN and DM2 were present in $39.9 \%$, $40.2 \%$ and $21 \%$ and $16.7 \%$ and $21.7 \%, 10.1 \%$, of males and females, respectively.

Table 3 depicts the histopathology characteristics of the LSG specimens. A total of $52 \%$ of the histopathology sample were normal. The most common abnormal histopathologies were chronic inactive gastritis (33\%), chronic active gastritis (6.8\%), follicular gastritis $(2.7 \%)$, lymphoid aggregates (2.2\%), intestinal metaplasia (1.4\%) and GIST (0.7\%). Rare histopathologies also existed, each comprising less than $0.2 \%$. We categorised the abnormal histopathologies into benign and pre-malignant conditions. Benign lesions included chronic inactive gastritis, chronic active gastritis and others (fundic gland polyp, lymphoplasmacytic non-caseating granuloma, submucosal fibrosis with eosinophil-rich chronic inflammation, focal gangrenous necrosis, mesothelial chronic inflammation, focal prominence of intramural neural tissue, pancreatic heterotopia, leiomyoma and gastric lipoma). Premalignant lesions included follicular gastritis, lymphoid aggregate, intestinal metaplasia, GIST and others (atrophic

Table 2 Demographic, anthropometric and clinical characteristics of LSG patients $(N=1555)$

\begin{tabular}{lll}
\hline Characteristic & Male & Female \\
& $N=471(30.3 \%)$ & $N=1084(69.7 \%)$ \\
\hline $\begin{array}{l}\text { Age (years) } \\
\text { M } \pm \text { SD }\end{array}$ & $35.3 \pm 11.4$ & $36 \pm 10.3$ \\
Range & $13-74$ & $14-65$ \\
BMI & & \\
$\mathrm{M} \pm \mathrm{SD}$ & $48 \pm 9.1$ & $46.3 \pm 8.1$ \\
Range & $25-72$ & $20-74$ \\
H. pylori & & \\
Yes (\%) & 39.9 & 40.2 \\
Hypertension & & \\
$\quad \begin{array}{l}\text { Yes (\%) } \\
\text { Diabetes mellitus } \\
\text { Yes (\%) }\end{array}$ & 21.0 & 16.7 \\
\hline
\end{tabular}

$M$ mean, $S D$ standard deviation chronic gastritis, autoimmune gastritis, dysplastic neuroendocrine nodule).

Table 4 shows the comparisons between the different benign and pre-malignant histopathologies with each other and with normal controls. In terms of BMI, for each of the five research questions that were examined, no significant associations were observed between the different types of histopathologies and the different control specimens.

Spearman correlation showed very weak correlations between BMI and the other covariates (age, gender, H. pylori, HTN, DM2, where correlation values ranged between 0.002 0.077). Exploratory linear regression analysis was undertaken to assess the predictive strength of BMI using the covariates. $R 2$ (coefficient of determination) value was less than 0.05 in most of the cases (data not presented).

\section{Discussion}

There exists a noticeable deficiency in the published literature that examined the relationships between different histopathologies of LSG specimens and BMI. In the current study, the BMI of patients with either benign or pre-malignant histopathologies in the LSG specimens was not significantly different when compared with the BMI of patients having other histopathologies or with BMI of controls with normal specimens (Table 4). Likewise, the BMI of patients with benign specimens (grouped together) was not significantly different than the BMI of controls with normal specimens. Similarly, the BMI of patients with pre-malignant specimens (grouped together) was not significantly different from the BMI of controls with normal specimens. In addition, the BMI of patients with benign lesions was not significantly different than the BMI of patients with pre-malignant lesions.

One study of five GIST cases reported significantly lower BMI for patients with GIST compared to patients without GIST histopathology [17]. In contrast, a study in USA found no significant difference between LSG specimens with abnormal pathology (i.e. not containing H. pylori or gastric metaplasia) vs. specimens with significant pathology (i.e. containing H. pylori or gastric metaplasia) [18]. Likewise, others reported no significant relationship between BMI and the presence or absence of gastric atrophy, H. pylori, lymphoid follicle and lymphoid aggregates [19]. An exception was intestinal metaplasia, which was associated with lower BMI (40.8) when compared to other abnormal specimens (atrophy, H. pylori, lymphoid follicle and lymphoid aggregate) that did not have intestinal metaplasia (BMI 44.9, $p<0.0001$ ) [19]. However, the current study observed that patients with GIST, intestinal metaplasia or lymphoid aggregates had generally higher BMI compared to patients without these particular conditions or compared to patients with normal histopathology, but the differences were not statistically significant. 
Table 3 Characteristics of LSG histopathology specimens $(N=$ 1555)

\begin{tabular}{|c|c|}
\hline Histopathology of LSG Specimen & $n(\%)$ \\
\hline Normal histopathology & $810(52)$ \\
\hline Abnormal histopathology & $745(48)$ \\
\hline Chronic inactive gastritis (mild or moderate) & $512(33)$ \\
\hline Chronic active gastritis & $105(6.8)$ \\
\hline Follicular gastritis & $43(2.7)$ \\
\hline Lymphoid aggregate & $35(2.2)$ \\
\hline Intestinal metaplasia & $22(1.4)$ \\
\hline GIST & $11(0.7)$ \\
\hline Fundic gland polyp & $3(0.19)$ \\
\hline Atrophic chronic gastritis & $3(0.19)$ \\
\hline Lymphoplasmacytic non-caseating granuloma & $2(0.13)$ \\
\hline Submucosal fibrosis with eosinophil-rich chronic inflammation & $1(0.06)$ \\
\hline Focal gangrenous necrosis & $1(0.06)$ \\
\hline Autoimmune gastritis & $1(0.06)$ \\
\hline Mesothelial chronic inflammation & $1(0.06)$ \\
\hline Focal prominence of intramural neural tissue & $1(0.06)$ \\
\hline Dysplastic neuroendocrine nodule & $1(0.06)$ \\
\hline Leiomyoma & $1(0.06)$ \\
\hline Pancreatic heterotopia & $1(0.06)$ \\
\hline Gastric lipoma & $1(0.06)$ \\
\hline All benign ${ }^{a}$ & $629(40.5)$ \\
\hline All pre-malignant ${ }^{b}$ & $116(7.5)$ \\
\hline
\end{tabular}

$L S G$ laparoscopic sleeve gastrectomy, GIST gastrointestinal stromal tumour. ${ }^{a}$ includes chronic inactive gastritis, chronic active gastritis and others (fundic gland polyp, lymphoplasmacytic non-caseating granuloma, submucosal fibrosis with eosinophil-rich chronic inflammation, focal gangrenous necrosis, mesothelial chronic inflammation, focal prominence of intramural neural tissue, pancreatic heterotopia, leiomyoma and gastric lipoma; ${ }^{b}$ includes follicular gastritis, lymphoid aggregate, intestinal metaplasia, GIST and others (atrophic chronic gastritis, autoimmune gastritis, dysplastic neuroendocrine nodule)
Likewise, in the current study, patients with follicular gastritis generally exhibited higher BMI than patients without such pathology or compared to patients with normal LSG specimens (BMI of follicular gastritis patients 48.3 vs. other histopathology 46.6 vs. normal 46.5 ), but again, and differences were not statistically significant (Table 4).

Such inconsistent findings across the various studies raise four interlacing issues: categorisation of groups of histopathology types, sample size, confounding and controls.

In terms of categorisation of groups of histopathology types, studies are inconsistent in the ways they categorise groups of histopathologies. Such undertaking renders the comparisons of the findings of different studies difficult and probably imprecise. For instance, one study categorised the histopathologies they found into LSG specimens with abnormal pathology (i.e. not containing H. pylori or gastric metaplasia) vs. specimen with significant pathology (containing H. pylori or gastric metaplasia) [18]. Whilst it is not clear why $H$. pylori (an infection) was classified as pathology, the result is that comparisons between studies may consequently lack precision. In contrast to Hansen et al. [18], the current study observed that $H$. pylori is a potential confounder rather than pathology and employed it as such in the analysis (detailed below). Future research would benefit from a standardisation of the ways employed to categorise groups of histopathologies for more meaningful comparisons.

As for the sample size, among the sparse literature that assessed the relation between the BMI and histopathology of LSG specimens, the current study's sample size was the largest (1555 patients) when compared to others, e.g. Adalı et al. (37 patients with pathology) [19], Hansen et al. (351 patients, but not entirely clear if all were with pathology) [18] or Yuval et al. (827 patients, 5 GIST patients vs. 822 control group not having GIST pathology) [17]. Such large sample size provided the current study the advantage of minimizing the chance of assuming a false premise as true [20].

In connection with confounding variables, the current study is unique in that it controlled for potential confounding variables (e.g. age, gender, H. pylori infection, DM, HTN) when examining the relationship between different histopathologies of LSG specimens and BMI. Most previous studies had not undertaken such controlled analysis. For instance, 
Table 4 Comparisons between BMI of cases vs. different controls

\begin{tabular}{|c|c|c|c|c|c|c|c|c|}
\hline \multirow[b]{2}{*}{ Histopathology } & \multirow[b]{2}{*}{$N$} & \multirow[b]{2}{*}{ BMI } & \multicolumn{2}{|c|}{ Controls (all other histopathologies) } & \multirow[t]{2}{*}{$P^{a}$} & \multicolumn{2}{|c|}{ Controls (normal) } & \multirow[t]{2}{*}{$P^{b}$} \\
\hline & & & $N$ & BMI & & $N$ & BMI & \\
\hline \multicolumn{9}{|l|}{ Benign lesions } \\
\hline Chronic inactive gastritis & 512 & $46.8 \pm 8.2$ & 233 & $46.2 \pm 7.9$ & 1.000 & 810 & $46.5 \pm 8.0$ & 1.000 \\
\hline Chronic active gastritis & 105 & $47.7 \pm 7.6$ & 640 & $46.4 \pm 8.2$ & 0.301 & 810 & $46.5 \pm 8.0$ & 0.372 \\
\hline Others benign & 12 & $41.8 \pm 10.5$ & 733 & $46.8 \pm 8.5$ & 0.811 & 810 & $46.5 \pm 8.0$ & 0.770 \\
\hline \multicolumn{9}{|l|}{ Pre-malignant lesions } \\
\hline Follicular gastritis & 43 & $48.3 \pm 10.9$ & 703 & $46.6 \pm 8.1$ & 1.000 & 810 & $46.5 \pm 8.0$ & 1.000 \\
\hline Lymphoid aggregate & 35 & $44.8 \pm 7.2$ & 710 & $46.7 \pm 8.1$ & 0.463 & 810 & $46.5 \pm 8.0$ & 0.583 \\
\hline Intestinal metaplasia & 22 & $44.5 \pm 8.6$ & 723 & $46.6 \pm 8.1$ & 0.688 & 810 & $46.5 \pm 8.0$ & 0.791 \\
\hline GIST & 11 & $43.2 \pm 8.1$ & 734 & $46.6 \pm 8.1$ & 0.510 & 810 & $46.5 \pm 8.0$ & 0.564 \\
\hline Others pre-malignant & 5 & $44.4 \pm 6.7$ & 740 & $46.8 \pm 8.6$ & 0.615 & 810 & $46.5 \pm 8.0$ & 0.637 \\
\hline All benign vs. all pre-malignant & $629^{c}$ & $47.0 \pm 8.4$ & $116^{d}$ & $45.7 \pm 9.0$ & 0.924 & - & - & - \\
\hline All benign vs. all normal & $629^{c}$ & $47.0 \pm 8.4$ & - & - & - & 810 & $46.5 \pm 8.0$ & 0.702 \\
\hline All pre-malignant vs. all normal & $116^{d}$ & $45.7 \pm 9.0$ & - & - & - & 810 & $46.5 \pm 8.0$ & 0.918 \\
\hline
\end{tabular}

Analysis controlled for age, gender, H. pylori infection, diabetes mellitus and hypertension; all BMI values expressed as mean \pm standard deviation; ${ }^{a}$ denotes the $p$ value of differences in the BMI of patients with specific abnormal specimens vs. controls with abnormal LSG specimens other than the given one; ${ }^{b}$ denotes the $p$ value of differences in the BMI of patients with abnormal LSG specimens vs. controls with normal specimens; ${ }^{c}$ includes all benign abnormal specimens; ${ }^{d}$ includes all pre-malignant abnormal specimens; an en dash denotes not applicable

with the exception of Hansen et al. [18] who controlled for gender, medical comorbidities and H. pylori (but not age) in their analysis, previous studies do not seem to have controlled for any confounding variables that could have influenced their findings. Yuval et al. [17] did not control for age, gender, diabetes mellitus and HTN in their examination of the BMI of patients with GIST histopathology vs. the BMI of patients with no GIST histopathology; Saafan et al. [10] did not control for age, gender and H. pylori, and Adalı et al. [19] did not control for age, gender and diabetes mellitus in their assessment of the BMI of patients with different histopathologies (gastritis, atrophy, H. pylori, intestinal metaplasia, lymphoid follicle and lymphoid aggregate). Confounding is a distortion (inaccuracy) in the estimated measure of association that occurs when the primary exposure of interest is mixed up with some other factor that is associated with the outcome [21]. Hence, such controlling for a range of important confounding variables as the current study undertook e.g. age, gender, H. pylori, DM and HTN is critical in order to assess the 'true' relationship between LSG histopathology and BMI.

As confounding is a situation in which the association between an exposure and outcome is distorted by the presence of another variable [22], confounders raise two critical points. The first is that research of the relationships between histopathologies of LSG specimens and BMI, e.g. [10, 17, 19] all did not control for age, gender and diabetes mellitus in their assessment of the BMI of patients of different histopathologies, despite that these studies, judging from our appraisal of these studies' findings, seem to have had the data necessary for such controlled analyses available. This suggests that bariatric researchers will need to have heightened awareness of potential confounders, to collect the necessary data, and more importantly, include it in the analyses as mandatory.

The second point is that, given that confounders are variables that have a relationship to the exposure [23] (in this case, possibly BMI) and are also independently associated with the outcome (abnormal LSG histopathology), it is critical that a suspected confounder's relationships with both exposure and outcome are first ascertained for the relationship under examination. We ascertained such relationships in the context of the associations that the current study examined. In terms of their independent association with the outcome (abnormal histopathology), older age was significantly associated with GIST and intestinal metaplasia [10, 24, 25]; females were significantly associated with chronic active gastritis [10]; there is evidence that $H$. pylori is significantly associated with each of follicular gastritis, lymphoid aggregates, gastritis and intestinal metaplasia [10, 25-27] and with GIST [10]; and diabetes mellitus was associated with gastritis [28]. Nevertheless, to the best of our knowledge, there is still no evidence of a significant association between hypertension and LSG histopathology. As for a potential confounder's association to the exposure under examination, BMI was significantly associated with HTN [29], DM2 [30], H. pylori [31], gender [32] and age [32]. The current study used multivariable regression analysis to control for such identified confounders during data analysis, as recommended by others [33]. The current study also acknowledges that as the number of confounders that 
can be controlled for simultaneously is limited (may lead to small numbers in some strata), regression is frequently employed to control for more than one confounder at the same time [23].

Finally, in terms of controls, few studies have emphasised the importance of the appropriate selection of control groups [34]. The question 'Compared to What?' is critical when comparator group/s are employed, and there has been calls regarding the consideration of appropriate comparator group/s in the context of the relationships between different LSG histopathologies and BMI [35]. The use of a comparative group is essential for valid study findings, and the choice of 'which comparator?' could influence the internal validity of a study. Others have noted the research that included a control group did not state the explicit principles employed in the selection of controls, e.g. how controls were selected and why specific controls were engaged, suggesting a lack of scientific approach in the choice of controls [34]. For instance, one study [19] compared between specific abnormal histopathology and all other histopathologies as control but did not seem to explicitly state why such controls (and not normal controls) were used. Often, controls might comprise an easily obtained convenience group that might not necessarily be in agreement with the principles of control selection, resulting in selection bias [34]. Hence, the current study made explicit the selection of a series of different controls appropriate to answer each of the five questions that the study examined (Table 1). The answer to all these five questions was that there were no significant differences in BMI between the groups when age, gender, $H$. pylori infection, DM and HTN were controlled for in Spearman's correlation and multivariable analysis.

Generally, pre-malignant lesions identified in LSG specimens across 11 studies varied from 4.1 to $33.2 \%$ $[10,18,36-44]$. However, across these 11 abovementioned studies, there was zero actual malignancy reported, despite that these studies were undertaken in a wide variety of geographical areas worldwide with different risk of gastric cancers. The lack of any malignancy in LSG specimens across these studies is in agreement with the findings of the current research. In addition, the literature on LSG specimens is certainly sparse on information about the definite risk of premalignant gastric lesions that actually turn malignant. Thus, even with our $7.5 \%$ pre-malignant lesions identified in LSG specimens, and with the huge number of LSG procedures undertaken worldwide, such current lack of the evidence base renders us unable to extrapolate in any way the potential number of patients with pre-malignant lesions that would actually turn malignant or whether such numbers would be high and alarming or otherwise. Hence, there exists much debate regarding the role of routine histopathological examination of LSG specimens $[10,18,36-44]$, and some authors have suggested that histopathological examination of LSG specimens is to be done when intraoperative macroscopic pathology is observed [10, 18, 43]. Future research would benefit from addressing these points.

This study has limitations. It is a retrospective study with its inherent limitations (e.g. potentially missing patients). Some of the pathologies had only a few or one case (e.g. gastric lipoma, leiomyoma, autoimmune gastritis) (Table 2), rendering comparisons not very reliable. The relationship between BMI and histopathology is not a causation but rather, is an association as it cannot be determined if exposure (BMI) preceded disease (histopathology). Patients with no $H$. pylori detected at preoperative OGD were not tested to assess if they had previous $H$. pylori infection that was eradicated but could have contributed to the current histopathology identified. Future research should try to overcome such limitations, particularly with employing larger number of patients with each abnormal histopathology in order to confirm or refute the findings of the current study.

\section{Conclusion}

In the current study, among the 1555 patients who had LSG, after controlling for confounding variables (age, gender, $H$. pylori, diabetes mellitus type 2, hypertension), there was no significant association between BMI and either the different benign or pre-malignant histopathologies identified in their gastric specimens. Moreover, there was a very weak correlation between BMI and the other covariates (age, gender, H. pylori, HTN, DM2).

Acknowledgements Open Access funding provided by the Qatar National Library. The authors wish to thank Dr. Prem Chandra for assistance with the statistical analysis and the Medical Research Center at HMC for their support for the current study.

\section{Compliance with Ethical Standards}

Conflict of Interest The authors declare that there are no sources or grants of financial support from any institution to be disclosed and no conflict of interests to be disclaimed or financial disclosure to be declared.

Statement of Informed Consent The informed consent was waived (IRB approved, HIPAA compliant retrospective study).

Statement of Human and Animal Rights All procedures performed in studies involving human participants were in accordance with the ethical standards of the institutional and/or national research committee and with the 1964 Helsinki declaration and its later amendments or comparable ethical standards. 
Open Access This article is distributed under the terms of the Creative Commons Attribution 4.0 International License (http:// creativecommons.org/licenses/by/4.0/), which permits unrestricted use, distribution, and reproduction in any medium, provided you give appropriate credit to the original author(s) and the source, provide a link to the Creative Commons license, and indicate if changes were made.

\section{References}

1. World Health Organization (2016). Obesity and overweight. [cited 2016 August 15]. Available from http://www.who.int/mediacentre/ factsheets/fs311/en/

2. Schirmer B, Schauer PR. The surgical management of obesity. In: Brunicardi F, Andersen D, Hunter J, et al., editors. Schwartz's principles of surgery ninth edition. USA: McGraw-Hill Companies; 2010. p. 952-3.

3. Setiawan VW, Yang HP, Pike MC, et al. Type I and II endometrial cancers: have they different risk factors? J Clin Oncol. 2013;31(20): 2607-18.

4. Dougan MM, Hankinson SE, Vivo ID, et al. Prospective study of body size throughout the life-course and the incidence of endometrial cancer among premenopausal and postmenopausal women. Int J Cancer. 2015;137(3):625-37.

5. Hoyo C, Cook MB, Kamangar F, et al. Body mass index in relation to oesophageal and oesophagogastric junction adenocarcinomas: a pooled analysis from the International BEACON Consortium. Int $\mathbf{J}$ Epidemiol. 2012;41(6):1706-18.

6. Chen Y, Liu L, Wang X, et al. Body mass index and risk of gastric cancer: a meta-analysis of a population with more than ten million from 24 prospective studies. Cancer Epidemiol Biomark Prev. 2013;22(8):1395-408.

7. Chen Y, Wang X, Wang J, et al. Excess body weight and the risk of primary liver cancer: an updated meta-analysis of prospective studies. Eur J Cancer. 2012;48(14):2137-45.

8. Campbell PT, Newton CC, Freedman ND, et al. Body mass index, waist circumference, diabetes, and risk of liver cancer for U.S. adults. Cancer Res. 2016;76(20):6076-83.

9. Wang $\mathrm{F}, \mathrm{Xu} \mathrm{Y}$. Body mass index and risk of renal cell cancer: a dose-response meta-analysis of published cohort studies. Int $\mathrm{J}$ Cancer 2014;135(7):1673-1686.

10. Saafan T, Bashah M, El Ansari W, et al. Erratum to: Histopathological changes in laparoscopic sleeve gastrectomy specimens: prevalence, risk factors, and value of routine histopathologic examination. Obes Surg. 2017;27(10):2778.

11. Siddiqui ST, Naz E, Danish F, et al. Frequency of Helicobacter pylori in biopsy proven gastritis and its association with lymphoid follicle formation. J Pak Med Assoc. 2011;61(2):138-41.

12. Ahmad A, GovilY FBB. Gastric mucosa-associated lymphoid tissue lymphoma. Am J Gastroenterol. 2003;98:975-86.

13. Zullo A, Hassan C, Romiti A, et al. Follow-up of intestinal metaplasia in the stomach: when, how and why. World J Gastrointest Oncol. 2012;4(3):30-6.

14. Park YH, Kim N. Review of atrophic gastritis and intestinal metaplasia as a premalignant lesion of gastric cancer. J Cancer Prev. 2015;20(1):25-40.

15. Bizzaro N, Antico A, Villalta D. Autoimmunity and gastric cancer. Int J Mol Sci. 2018;19(2):377.

16. Ishida M, Sekine S, Fukagawa T, et al. Neuroendocrine carcinoma of the stomach: morphologic and immunohistochemical characteristics and prognosis. Am J Surg Pathol. 2013;37(7):949-59.
17. Yuval JB, Khalaileh A, Abu-Gazala M, et al. The true incidence of gastric GIST-a study based on morbidly obese patients undergoing sleeve gastrectomy. Obes Surg. 2014;24(12):2134-7.

18. Hansen SK, Pottorf BJ, Hollis Jr HW, et al. Is it necessary to perform full pathologic review of all gastric remnants following sleeve gastrectomy? Am J Surg. 2017;214(6):1151-5.

19. Adalı Y, Binnetoğlu K, Eroğlu HA, et al. The relationship between histopathologic findings and body mass index in sleeve gastrectomy materials. Obes Surg. 2018; https://doi.org/10.1007/s11695018-3518-z.

20. Faber J, Fonseca LM. How sample size influences research outcomes. Dental Press J Orthod. 2014;19(4):27-9.

21. LaMorte W, Sullivan L (2016, June 3). Confounding and effect measure modification. Retrieved December 26, 2018, from http:// sphweb.bumc.bu.edu/otlt/MPH-Modules/BS/BS704-EP713 Confounding-EM/

22. Onlinecourses.science.psu.edu. (2018). 3.5 - Bias, Confounding and Effect Modification | STAT 507. [online] Available at: https:// onlinecourses.science.psu.edu/stat507/node/34/ [Accessed 28 Nov. 2018].

23. Health Knowledge. (2018). Confounding in epidemiological studies. [online] Available at: https://www.healthknowledge.org. uk/node/803 [Accessed 28 Nov. 2018].

24. Tran T, Davila JA, El-Serag HB. The epidemiology of malignant gastrointestinal stromal tumors: an analysis of 1,458 cases from 1992 to 2000. Am J Gastroenterol. 2005;100:162-8.

25. Leung WK, Ng EK, Chan WY, et al. Risk factors associated with the development of intestinal metaplasia in first-degree relatives of gastric cancer patients. Cancer Epidemiol Biomark Prev. 2005; 14(12):2982-6.

26. Chen XY, Liu WZ, Shi Y, et al. Helicobacter pylori associated gastric diseases and lymphoid tissue hyperplasia in gastric antral mucosa. J Clin Pathol. 2002;55(2):133-7.

27. Kara N, Urganci N, Kalyoncu D, et al. The association between Helicobacter pylori gastritis and lymphoid aggregates, lymphoid follicles and intestinal metaplasia in gastric mucosa of children. $\mathrm{J}$ Paediatr Child Health. 2014;50(8):605-9.

28. Boehme MW, Autschbach F, Ell C, et al. Prevalence of silent gastric ulcer, erosions or severe acute gastritis in patients with type 2 diabetes mellitus - a cross-sectional study. Hepatogastroenterology. 2007;54:643-8.

29. Roka R, Michimi A, Macy G. Associations between hypertension and body mass index and waist circumference in U.S. adults: a comparative analysis by gender. High Blood Press Cardiovasc Prev. 2015;22(3):265-73.

30. Ganz ML, Wintfeld N, Li Q, et al. The association of body mass index with the risk of type 2 diabetes: a case-control study nested in an electronic health records system in the United States. Diabetol Metab Syndr. 2014;6(1):50.

31. Suki M, Leibovici Weissman Y, Boltin D, et al. Helicobacter pylori infection is positively associated with an increased BMI, irrespective of socioeconomic status and other confounders: a cohort study. Eur J Gastroenterol Hepatol. 2018;30(2):143-8.

32. Nevill AM, Metsios GS. The need to redefine age- and genderspecific overweight and obese body mass index cutoff points. Nutr Diabetes. 2015;5(11):e186.

33. Pannucci CJ, Wilkins EG. Identifying and avoiding bias in research. Plast Reconstr Surg. 2010;126(2):619-25.

34. Malay S, Chung KC. The choice of controls for providing validity and evidence in clinical research. Plast Reconstr Surg. 2012;130(4): 959-65.

35. Saafan T, El Ansari W. Sorting Out the Myths from the Facts: Commentary on Yasemen Adali et al. The Relationship Between Histopathologic Findings and Body Mass Index in Sleeve Gastrectomy Materials. Obes Surg. (2018) https://doi.org/10. 1007/s11695-018-03693-4. 
36. AbdullGaffar B, Raman L, Khamas A, et al. Should we abandon routine microscopic examination in bariatric sleeve gastrectomy specimens? Obes Surg. 2016;26(1):105-10.

37. Clapp B. Histopathologic findings in the resected specimen of a sleeve gastrectomy. JSLS. 2015;19(1):e2013.00259. https://doi. org/10.4293/JSLS.2013.00259.

38. Raess PW, Baird-Howell M, Aggarwal R, et al. Vertical sleeve gastrectomy specimens have a high prevalence of unexpected histopathologic findings requiring additional clinical management. Surg Obes Relat Dis. 2015;11(5):1020-3.

39. Almazeedi S, Al-Sabah S, Al-Mulla A, et al. Gastric histopathologies in patients undergoing laparoscopic sleeve gastrectomies. Obes Surg. 2013;23:314-9.

40. Lauti M, Gormack SE, Thomas JM, et al. What does the excised stomach from sleeve gastrectomy tell us? Obes Surg. 2016;26(4): $839-42$.

41. Ohanessian SE, Rogers AM, Karamchandan DM. Spectrum of gastric histopathologies in severely obese American patients undergoing sleeve gastrectomy. Obes Surg. 2016;26(3):595-602.
42. Canil AM, Iossa A, Termine P, et al. Histopathology findings in patients undergoing laparoscopic sleeve gastrectomy. Obes Surg. 2018;28(6):1760-5.

43. Walędziak M, Różańska-Walędziak A, Janik MR, et al. Macroscopic evaluation of gastric specimens after laparoscopic sleeve gastrectomy-an optimum screening test for incidental pathologies? Obes Surg. 2019;29(1):28-31. https://doi.org/10.1007/ s11695-018-3485-4.

44. Yardimci E, Bozkurt S, Baskoy L, et al. Rare entities of histopathological findings in 755 sleeve gastrectomy cases: a synopsis of preoperative endoscopy findings and histological evaluation of the specimen. Obes Surg. 2018;28(5):1289-95.

Publisher's Note Springer Nature remains neutral with regard to jurisdictional claims in published maps and institutional affiliations. 